Original Research Paper

\title{
Speed of Sound and Apparent Molal Volume Approach to Study the Ion-Solvent Interactions in Pharmaceutically Important Liquid Systems
}

\author{
${ }^{1}$ Ganesh, D.N., ${ }^{2}$ Vinay S. Bhat and ${ }^{2}$ Susmita Kamila \\ ${ }^{l}$ Department of Chemistry, Rajiv Gandhi Institute of Technology (VTU), Bangalore - 32, India \\ ${ }^{2}$ Department of Chemistry, East Point College of Engineering and Technology (VTU), Bangalore -49, India
}

Article history

Received: 17-02-2020

Revised: 14-04-2020

Accepted: 20-04-2020

Corresponding Author:

Susmita Kamila

Department of Chemistry, East

Point College of Engineering

and Technology, Bangalore -

49, India

Bhubaneswar, Odisha.

Email:sushkam@yahoo.co.in

\begin{abstract}
Presence of zinc ion and lactose in different pharmaceutical compositions have been inspired to investigate the ion-solvation and related interactions among the components in solution media. This study has been carried out by the measurements of ultrasonic velocity, viscosity and density for the binary solutions of zinc sulphate $\left(\mathrm{ZnSO}_{4}\right)$ in lactose-water mixed solvent systems at 303.15, 308.15, 313.15 K temperature and at atmospheric pressure. Different thermo-acoustic parameters such as isentropic compressibility, apparent molal volume, solvation number, free volume, internal pressure etc., have been evaluated from the measured values. All these parameters have been analyzed in the light of molecular interactions from their variations against concentration as well as temperature. Presence of ion-solvation and structure promoting nature of solute are the major findings in the present investigation. Besides, it also reveals that the ion-solvation decreases as the temperature increases.
\end{abstract}

Keywords: Solute-Solvent Interaction, Ion-Solvation, Thermo-Acoustic Parameters, Apparent Molal Compressibility

\section{Introduction}

Certain metal ions are essential for different types of critical functions in human organs. Scarcity of such metal ions can lead to many complicated diseases. Few alkali metals including some transition metals are currently known to be required for normal biological functions in humans and even other living organisms (Lane and Morel, 2006; Staiger and Pietak, 2006). Zinc is an essential and most important trace element in biological systems (Coleman, 1967). It is the second most abundant transition metal in organisms after iron and it is the only metal which appears in all enzyme classes (Wapnir, 1990; Broadley et al., 2007). Zinc metal is mostly present in the brain, muscle, bones, kidney and liver (Pfeiffer and Braverman, 1982). Deficiency of zinc produces growth retardation, skin lesions, poor appetite and loss of body hair etc. Even the deficiency of this metalion affects adversely chronic diseases like neurological disorders, Alzheimer's disease and other age-related diseases (Evans, 1986). The unique properties of zinc may have significant therapeutic benefits in these diseases. Besides, zinc ions along with other metal ions such as magnesium, potassium, sodium etc., have their importance in pharmaceutics for the preparations of various drugs. Especially, zinc sulfate is used to treat and to prevent zinc deficiency and also it is used as an alternative to, or in a rotation with, copper sulphate for controlling digital dermatitis. It is supposed to be a better supplement for chronic diarrhea (Khan and Sellen, 2011).

In continuation of our earlier works on ion solvation (Kamila et al., 2017; Dash and Kamila, 2017), presently the inter-molecular interactions of zinc sulphate are studied with a saccharide like lactose in water systems. Generally, carbohydrates are supposed to be the major component of the entire living organisms in energy production and other protein synthesis processes. Various saccharides are known to have their functions as stabilizing agents for the native state of proteins and enzymes (Dash and Kamila, 2015; Kannappan and Vinayagam, 2006). They also show their importance in the field of chemicals, bio-chemicals, foods and pharmaceutical industries. Besides, it is exclusively present in milk apart from medicines and culture media. Absorption of lactose is dependent upon the enzyme 
lactase that enhances the absorption of calcium, magnesium and zinc. It also promotes the growth of lactobacilli and provides galactose, which is essential for the formation of cerebral galactolipids and hence development of the brain. Review of literature shows that zinc sulphate mixed with lactose of different potencies is used in homeopathy for the biological activity (Chauhan et al., 2013).

Number of studies has been reported for various electrolytes with mixed solvent systems for the intermolecular association studies (Kamila et al., 2017; Dash and Kamila, 2017; 2015; Kannappan and Vinayagam, 2006; Jones and Dole, 1929; Nain et al., 2013a). Besides, interaction studies have also been reported using different carbohydrates as major reacting components in the binary systems (Gupta, 1991; Timasheff and Arakawa, 1989; Nithiyanantham and Palaniappan, 2013; Kannappan and Palani, 2007). But, however, studies involving transition metal ion with a disaccharide in mixed solvent system is scarce in literature. So an attempt has been made to study the solvation of zinc sulphate in lactose-water mixed solvent system at three different temperatures.

\section{Material and Methods}

Analytical grade zinc sulphate heptahydrate and lactose were procured from Qualigen Chemicals (more than $99.9 \%$ purity). Stock solutions of lactose were prepared by taking accurate weight of solute in freshly prepared double distilled water (w/V ratio). The waterlactose mixed solvent system varies from $0 \%$ to $10 \%$ (w/V). Similarly, different $\mathrm{ZnSO}_{4}$ solutions in lactosewater mixed solvents were prepared by taking exact amount of $\mathrm{ZnSO}_{4}$ in mixed solvents and the concentration of zinc sulphate varies from 0.01 to 0.1 mole $\mathrm{dm}^{-3}$. The solutions were kept for half an hour in a thermostat to acquire different temperature with an accuracy of $0.01 \mathrm{~K}$. The densities of all solutions were measured by a bicapillary pyknometer with deionized double distilled water with $0.9960 \times 10^{3} \mathrm{~kg} \mathrm{~m}^{-3}$ as its density at different temperatures. The precision of density measurement was within $\pm 0.0003 \mathrm{~kg} \mathrm{~m}^{-3}$. The viscosity measurements of the solutions were carried out by using a calibrated Ostwald viscometer. The constant temperature was maintained by immersing the viscometer in a water bath maintained within $\pm 0.01 \mathrm{~K}$. The ultrasonic velocity of all the solutions were measured at $303.15 \mathrm{~K}, 308.15 \mathrm{~K}, 313.15 \mathrm{~K}$ temperature and atmospheric pressure by a Mittal make (India) singlecrystal variable-path ultrasonic interferometer operating at $3 \mathrm{MHz}$ by circulating water from a thermostatically regulated bath maintained within $\pm 0.01 \mathrm{~K}$ around the sample holder for constant temperature. The velocity measurements were précised to $\pm 0.5 \mathrm{~ms}^{-1}$.

\section{Results}

These measured values of ultrasonic velocity, density and viscosity were used to evaluate different acoustic parameters such as isentropic compressibility, $\beta_{s}$ and intermolecular free length, $L_{f}$, using standard formulae (Franks et al., 1972; Kaulgud, 1976; Kamila et al., 2005). In addition, parameters like free volume, $V_{f}$, internal pressure, $\pi_{i}$, relaxation time, $\tau$ and relative association, $R_{A}$ were calculated using following relations (Kamila et al., 2017):

$$
\begin{aligned}
& V_{f}=\left(M_{e f f} U / K\right)^{3 / 2} \\
& \pi_{i}=b R T\left(\frac{K}{U}\right)^{\frac{1}{2}} \frac{\rho^{2 / s}}{M_{e f f}^{7 / 6}} \\
& \tau=4 / 3 \rho U^{2} \\
& R A=\left(\rho / \rho_{0}\right)\left(\frac{U_{o}}{U}\right)^{\frac{1}{3}}
\end{aligned}
$$

where, $b$ is the space packing factor generally 2 for liquids, $R$ is the gas constant, $T$ is absolute temperature and $K$ is a constant equal to $4.28 \times 10^{9}$, independent of temperature for all types of liquids. $M_{\text {eff }}$ is the effective molecular mass. Similarly, Parameters like apparent molal volume, $\phi_{v}$ and apparent molal compressibility, $\phi_{k}$ can be determined by using the following formulae (Kamila and Dash, 2012; Kamila et al., 2004; Nain et al., 2013b; Wadi and Ramasami, 1997):

$\phi_{v}=\frac{1000\left(\rho_{0}-\rho\right)}{c \rho \rho_{0}}+\frac{M}{\rho}$
$\phi_{k}=\frac{1000\left(\beta_{s} \rho_{0}-\beta_{s}^{0} \rho\right)}{c \rho \rho_{0}}+\frac{\beta_{s} M}{\rho}$

where, $\rho_{0}$ and $\beta_{s}^{0}$ are the density and isentropic compressibility of the solvent (aqueous-lactose), $\rho$ and $\beta_{s}$ are those of solution, respectively. $c$ is the molarity of the solution, $M$ is the molar mass of the solute (zinc sulphate), $n_{0}$ and $n_{i}$ are the moles of solvent and solute, respectively.

The limiting apparent molal volume $\phi_{v}^{0}$ was obtained using the method of linear regression from Masson's equation (Masson, 1929) based on Debye- Huckel theory as follows (Debye and Huckel, 1923):

$\phi_{v}=\phi_{v}^{0}+S_{v} c^{1 / 2}$

where, $\phi_{v}^{0}$ and $S_{v}$ are the intercept and slope in the plot of $\phi_{v}$ against $c^{1 / 2}$. Similarly, the limiting apparent molal 
compressibility, $\phi_{k}^{0}$, is obtained from Gucker's limiting law (Gucker Jr., 1933), which states $\phi_{k}$ as:

$\phi_{k}=\phi_{k}^{0}+S_{k} c^{1 / 2}$

where, $S_{k}$ is the slope related to ion-ion interaction and $\phi_{k}^{0}$ is the intercept related to ion-solvent interaction. The slope and intercept are obtained from the plot of $\phi_{k}$ against $c^{1 / 2}$. Solvation number, $S_{n}$, suggested by Passynski (1938) was calculated to assess the mode of association:

$S_{n}=\frac{n_{0}}{n_{i}}\left(1-\frac{\beta_{s}}{\beta_{s}^{0}}\right)$

where, $n_{0}$ and $n_{i}$ are the moles of solvent and solute, respectively. The limiting solvation number, $S_{n}^{0}$, is determined from the variation of the solvation number with molar concentration of solute. It can be calculated from the following relation:

$\lim _{c \rightarrow 0} \phi_{k}^{0}=-S_{n}^{0} V_{1}^{0} \beta_{s}^{0}$

where, $V_{1}^{0}$ is the molar volume of the solvent having moles of solvent as $n_{1}$.
The measured values of density, viscosity, ultrasonic velocity and computed values of other relevant acoustic parameters of zinc sulphate in water and different proportions of lactose-water systems at $303.15 \mathrm{~K}, 308.15 \mathrm{~K}$ and $313.15 \mathrm{~K}$ have been summarized in Table 1 to 5 whereas some parameters are graphically represented as figures.

\section{Discussion}

From the observations obtained in the present study, it reveals that the sound velocity, density and viscosity increase with the increase in the concentration of both zinc sulphate and lactose. As the concentration increases, the number of molecules in the medium increases. This makes the medium denser and reduces compressibility resulting in the increment of the sound velocity. The velocity was also found to increase with temperature indicating the structural rearrangement due to solvation (Singh and Banipal, 2008; Palani and Jayachitra, 2008). According to Thirumaran and Kannappan (2009), the increase in ultrasonic velocity with solute concentration and temperature is due to the cohesion brought about by ionic hydration. The increase in components increases the frictional resistance between the layers of the liquids and hence there is enhancement of coefficient of viscosity (Table 1). This decreases with temperature due to reduction in frictional forces among the particles.

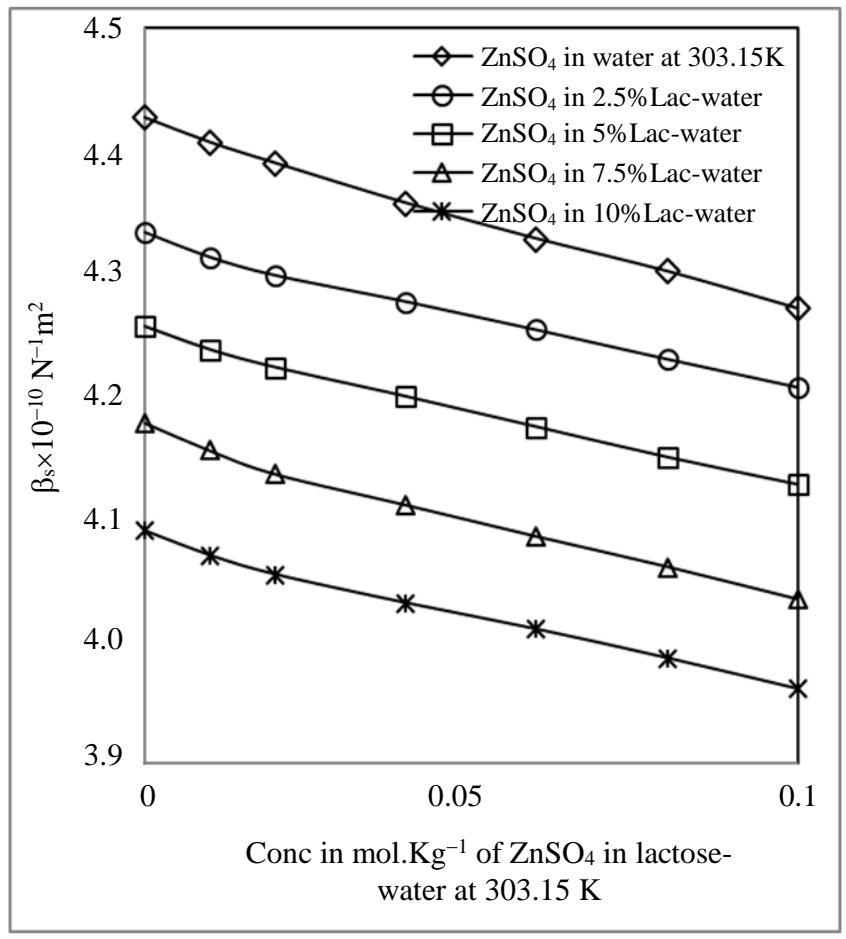

Fig. 1: Variation of isentropic compressibility, $\beta_{s} v s$. Concentration of $\mathrm{ZnSO}_{4}$ in water and Lactose-water mixed solvent at $303.15 \mathrm{~K}$ 
Table 1: Experimentally determined density, $\rho$, viscosity, $\eta$ and ultrasonic velocity, $\mathrm{U}$ of $\mathrm{ZnSO}_{4}$ in water, $2.5 \%, 5.0 \%, 7.5 \%$ and $10 \%$ lactose-water mixed solvent at $303.15,308.15$ and $313.15 \mathrm{~K}$ temperature

\begin{tabular}{|c|c|c|c|c|c|c|c|c|c|}
\hline \multirow[b]{2}{*}{$\mathrm{C}$ mol.kg ${ }^{-1}$} & \multicolumn{3}{|c|}{$\rho \times 10^{-3} \mathrm{~kg} \cdot \mathrm{m}^{-3}$} & \multicolumn{3}{|c|}{$\eta \times 10^{3} \mathrm{~kg} \cdot \mathrm{m}^{-1} \cdot \mathrm{s}^{-1}$} & \multicolumn{3}{|c|}{$\mathrm{U} \mathrm{m} \cdot \mathrm{s}^{-1}$} \\
\hline & $303.15 \mathrm{~K}$ & $308.15 \mathrm{~K}$ & $313.15 \mathrm{~K}$ & $303.15 \mathrm{~K}$ & $308.15 \mathrm{~K}$ & $313.15 \mathrm{~K}$ & $303.15 \mathrm{~K}$ & $308.15 \mathrm{~K}$ & $313.15 \mathrm{~K}$ \\
\hline \multicolumn{10}{|c|}{$\mathrm{ZnSO}_{4}$ in Water } \\
\hline 0.00 & 0.9964 & 0.9950 & 0.9937 & 0.7975 & 0.7234 & 0.6610 & 1505.8 & 1517.1 & 1525.4 \\
\hline 0.01 & 0.9987 & 0.9973 & 0.9959 & 0.8054 & 0.7307 & 0.6663 & 1507.5 & 1517.3 & 1527.1 \\
\hline 0.02 & 1.0008 & 0.9995 & 0.9976 & 0.8091 & 0.7390 & 0.6709 & 1508.8 & 1518.7 & 1529.7 \\
\hline 0.04 & 1.0044 & 1.0024 & 1.0009 & 0.8185 & 0.7474 & 0.6801 & 1511.7 & 1522.0 & 1534.7 \\
\hline 0.06 & 1.0073 & 1.0045 & 1.0040 & 0.8285 & 0.7549 & 0.6890 & 1514.6 & 1525.1 & 1537.8 \\
\hline 0.08 & 1.0107 & 1.0070 & 1.0062 & 0.8399 & 0.7647 & 0.6956 & 1516.7 & 1528.8 & 1539.7 \\
\hline 0.10 & 1.0136 & 1.0096 & 1.0089 & 0.8490 & 0.7729 & 0.7034 & 1519.9 & 1532.1 & 1542.0 \\
\hline \multicolumn{10}{|c|}{$\mathrm{ZnSO}_{4}$ in $2.5 \%$ Lactose-Water } \\
\hline 0.00 & 1.0060 & 1.0046 & 1.0030 & 0.8482 & 0.7672 & 0.6986 & 1514.7 & 1523.2 & 1533.1 \\
\hline 0.01 & 1.0079 & 1.0066 & 1.0050 & 0.8557 & 0.7747 & 0.7057 & 1516.8 & 1525.2 & 1534.8 \\
\hline 0.02 & 1.0096 & 1.0085 & 1.0065 & 0.8624 & 0.7817 & 0.7127 & 1518.1 & 1527.0 & 1536.5 \\
\hline 0.04 & 1.0129 & 1.0117 & 1.0096 & 0.8782 & 0.7907 & 0.7214 & 1519.4 & 1530.5 & 1538.2 \\
\hline 0.06 & 1.0162 & 1.0151 & 1.0123 & 0.8884 & 0.8000 & 0.7311 & 1521.0 & 1532.1 & 1541.1 \\
\hline 0.08 & 1.0191 & 1.0184 & 1.0152 & 0.8980 & 0.8089 & 0.7395 & 1523.1 & 1534.0 & 1542.6 \\
\hline 0.10 & 1.0222 & 1.0214 & 1.0181 & 0.9133 & 0.8187 & 0.7489 & 1524.9 & 1536.0 & 1544.8 \\
\hline \multicolumn{10}{|c|}{$\mathrm{ZnSO}_{4}$ in $5 \%$ Lactose-Water } \\
\hline 0.00 & 1.0151 & 1.0143 & 1.0125 & 0.8993 & 0.8118 & 0.7423 & 1521.4 & 1533.0 & 1540.9 \\
\hline 0.01 & 1.0172 & 1.0164 & 1.0145 & 0.9070 & 0.8253 & 0.7495 & 1523.2 & 1534.6 & 1542.7 \\
\hline 0.02 & 1.0192 & 1.0181 & 1.0163 & 0.9216 & 0.8335 & 0.7578 & 1524.3 & 1536.4 & 1544.3 \\
\hline 0.04 & 1.0224 & 1.0215 & 1.0199 & 0.9364 & 0.8484 & 0.7663 & 1526.2 & 1538.6 & 1547.2 \\
\hline 0.06 & 1.0259 & 1.0249 & 1.0234 & 0.9527 & 0.8641 & 0.7820 & 1528.1 & 1540.0 & 1549.2 \\
\hline 0.08 & 1.0286 & 1.0278 & 1.0266 & 0.9632 & 0.8729 & 0.7966 & 1530.6 & 1542.2 & 1551.4 \\
\hline 0.10 & 1.0309 & 1.0293 & 1.0281 & 0.9763 & 0.8867 & 0.8166 & 1533.0 & 1544.6 & 1553.6 \\
\hline \multicolumn{10}{|c|}{$\mathrm{ZnSO}_{4}$ in $7.5 \%$ Lactose-Water } \\
\hline 0.00 & 1.0238 & 1.0218 & 1.0197 & 0.9633 & 0.8678 & 0.7908 & 1529.0 & 1540.0 & 1547.0 \\
\hline 0.01 & 1.0262 & 1.0241 & 1.0220 & 0.9720 & 0.8762 & 0.7948 & 1531.4 & 1542.0 & 1548.8 \\
\hline 0.02 & 1.0284 & 1.0262 & 1.0242 & 0.9865 & 0.8845 & 0.8077 & 1533.3 & 1543.8 & 1550.2 \\
\hline 0.04 & 1.0323 & 1.0302 & 1.0284 & 1.0028 & 0.8938 & 0.8285 & 1535.1 & 1544.9 & 1553.0 \\
\hline 0.06 & 1.0353 & 1.0341 & 1.0322 & 1.0174 & 0.9088 & 0.8380 & 1537.7 & 1547.2 & 1554.7 \\
\hline 0.08 & 1.0385 & 1.0369 & 1.0352 & 1.0279 & 0.9186 & 0.8475 & 1540.0 & 1549.2 & 1557.6 \\
\hline 0.10 & 1.0416 & 1.0391 & 1.0376 & 1.0500 & 0.9346 & 0.8572 & 1542.8 & 1552.4 & 1560.0 \\
\hline \multicolumn{10}{|c|}{$\mathrm{ZnSO}_{4}$ in $10 \%$ Lactose-Water } \\
\hline 0.00 & 1.0327 & 1.0311 & 1.0302 & 1.0411 & 0.9324 & 0.8434 & 1538.8 & 1546.1 & 1555.9 \\
\hline 0.01 & 1.0356 & 1.0334 & 1.0325 & 1.0497 & 0.9408 & 0.8517 & 1540.4 & 1548.2 & 1557.6 \\
\hline 0.02 & 1.0370 & 1.0356 & 1.0345 & 1.0585 & 0.9494 & 0.8601 & 1542.3 & 1549.7 & 1559.1 \\
\hline 0.04 & 1.0404 & 1.0395 & 1.0383 & 1.0743 & 0.9654 & 0.8754 & 1544.2 & 1553.1 & 1562.0 \\
\hline 0.06 & 1.0435 & 1.0421 & 1.0413 & 1.0892 & 0.9771 & 0.8843 & 1546 & 1555.3 & 1563.8 \\
\hline 0.08 & 1.0468 & 1.0455 & 1.0446 & 1.1064 & 0.9902 & 0.8999 & 1548.1 & 1557.4 & 1565.6 \\
\hline 0.10 & 1.0495 & 1.0477 & 1.0467 & 1.1235 & 1.0018 & 0.9164 & 1551.0 & 1559.0 & 1569.0 \\
\hline
\end{tabular}

Isentropic compressibility, $\beta s$, an acoustic parameter, derived from the velocity data is known to be a sensitive indicator of the elasticity as well as of the molecular interactions of the system (Katrinak et al., 2012). In the present study, the compressibility decreases as the concentration of both zinc sulphate and lactose increases (Fig. 1). When the solute ion enters into the bulk of the solvent, the solvent molecules are attracted towards this ion because of electrostrictive force resulting in the decrease of solvent molecules for the incoming ions. This condition of the system is compression. Therefore, compressibility decreases with the increase in volumetric concentration of the solution. Moreover, there is breakage in the molecular structure of water with the addition of solute and making a more compact structure with the water molecules surrounded by the solute in mixed solvent. Santosh et al. (2011) also have reported similar type of observations and which was depicted as the existence of strong solutesolvent interactions through dipole-dipole and acceptor-donor interactions (Rao and Verrall, 1987; Nikam et al., 2000). This behaviour increases the inter molecular association and decreases $\beta s$ (Savaroglu and Ozdemir, 2008; Fort and Moore, 1965). There is also decrease in compressibility with temperature due to thermal expansion of the liquid systems (Fig. 2).

This compactness among the particles also reduces the intermolecular free length. According to literature, such type of behaviour is indicative of structurepromoting tendency of added solute as well as presence 
of significant ion-solvent interaction (Dash and Kamila, 2017; Kannappan and Vinayagam, 2007).

The apparent molal compressibility shows negative variation through out the concentration range indicating specific interaction involving ion-solvent type (Fig. 3 and 4) (Palani et al., 2008). According to Dhanalakshmi and Vasantharani (1999), the negative values of apparent compressibility are indicative of electrostrictive solvation of ions. The limiting apparent compressibility $\phi_{k}^{0}$ is the compressibility of solute at infinite dilution and it reflects the effects of solute-solvent interactions. The negative variation of this parameter indicates ion- solvent interaction and positive values of $\mathrm{S}_{\mathrm{k}}$ represent ion-ion interaction (Pal and Chauhan, 2011).

The dissociation occurs during the mixing of components resulting in the increases of free volume whereas association between the components will reduce the free volume. So the decreased values of free volume with the lactose concentration in the present study (Fig. 5) suggests the association due to $\mathrm{H}$ bonding with the water molecules (Table 2). Again with the increase in solute concentration the strength of interaction among the components increases and as a result there would be decrease in free volume.

Table 2: Calculated values of isentropic compressibility, $\beta_{\mathrm{s}}$, intermolecular free length, $\mathrm{Lf}$, free volume, $\mathrm{V}_{\mathrm{f}}$, of $\mathrm{ZnSO}_{4}$ in water, $2.5 \%, 5.0 \%, 7.5 \%$ and $10 \%$ lactose-water mixed solvent at $303.15,308.15$ and $313.15 \mathrm{~K}$ temperature

\begin{tabular}{|c|c|c|c|c|c|c|c|c|c|}
\hline \multirow[b]{2}{*}{$\mathrm{C}$ mol. $\mathrm{kg}^{-1}$} & \multicolumn{3}{|c|}{$\beta_{\mathrm{s}} \times 10^{10} \mathrm{~N}^{-1} \cdot \mathrm{m}^{2}$} & \multicolumn{3}{|c|}{$\mathrm{L}_{\mathrm{f}} \times 10^{11} \mathrm{M}$} & \multicolumn{3}{|c|}{$\mathrm{V}_{\mathrm{f}} \times 10^{8} \mathrm{~m}^{3} \cdot \mathrm{mol}^{-1}$} \\
\hline & $303.15 \mathrm{~K}$ & $308.15 \mathrm{~K}$ & $313.15 \mathrm{~K}$ & $303.15 \mathrm{~K}$ & $308.15 \mathrm{~K}$ & $313.15 \mathrm{~K}$ & $303.15 \mathrm{~K}$ & $308.15 \mathrm{~K}$ & $313.15 \mathrm{~K}$ \\
\hline \multicolumn{10}{|c|}{$\mathrm{ZnSO}_{4}$ in Water } \\
\hline 0.00 & 4.4262 & 4.3667 & 4.3249 & 4.3655 & 4.3778 & 4.3984 & 2.2407 & 2.6228 & 3.0276 \\
\hline 0.01 & 4.4061 & 4.3554 & 4.3058 & 4.3556 & 4.3722 & 4.3887 & 2.2205 & 2.5947 & 3.0087 \\
\hline 0.02 & 4.3892 & 4.3378 & 4.2838 & 4.3472 & 4.3634 & 4.3775 & 2.2171 & 2.5650 & 2.9976 \\
\hline 0.04 & 4.3567 & 4.3066 & 4.2419 & 4.3311 & 4.3476 & 4.3560 & 2.2030 & 2.5507 & 2.9754 \\
\hline 0.06 & 4.3276 & 4.2801 & 4.2118 & 4.3166 & 4.3342 & 4.3405 & 2.1871 & 2.5410 & 2.9507 \\
\hline 0.08 & 4.3011 & 4.2488 & 4.1922 & 4.3034 & 4.3184 & 4.3304 & 2.1646 & 2.5218 & 2.9380 \\
\hline 0.10 & 4.2707 & 4.2197 & 4.1685 & 4.2881 & 4.3035 & 4.3182 & 2.1535 & 2.5096 & 2.9187 \\
\hline \multicolumn{10}{|c|}{$\mathrm{ZnSO}_{4}$ in $2.5 \%$ Lactose-Water } \\
\hline 0.00 & 4.3326 & 4.2904 & 4.2419 & 4.3191 & 4.3394 & 4.3560 & 2.1361 & 2.5043 & 2.9104 \\
\hline 0.01 & 4.3125 & 4.2706 & 4.2241 & 4.3090 & 4.3294 & 4.3469 & 2.1211 & 2.4829 & 2.8830 \\
\hline 0.02 & 4.2978 & 4.2525 & 4.2084 & 4.3017 & 4.3202 & 4.3388 & 2.1076 & 2.4639 & 2.8569 \\
\hline 0.04 & 4.2765 & 4.2197 & 4.1862 & 4.2910 & 4.3035 & 4.3274 & 2.0703 & 2.4501 & 2.8330 \\
\hline 0.06 & 4.2537 & 4.1968 & 4.1594 & 4.2796 & 4.2918 & 4.3135 & 2.0545 & 2.4308 & 2.8075 \\
\hline 0.08 & 4.2299 & 4.1728 & 4.1394 & 4.2676 & 4.2796 & 4.3031 & 2.0423 & 2.4147 & 2.7864 \\
\hline 0.10 & 4.2071 & 4.1497 & 4.1159 & 4.2561 & 4.2677 & 4.2908 & 2.0105 & 2.3949 & 2.7617 \\
\hline \multicolumn{10}{|c|}{$\mathrm{ZnSO}_{4}$ in $5 \%$ Lactose-Water } \\
\hline 0.00 & 4.2560 & 4.1952 & 4.1596 & 4.2808 & 4.2910 & 4.3136 & 2.0420 & 2.4083 & 2.7760 \\
\hline 0.01 & 4.2372 & 4.1778 & 4.1418 & 4.2713 & 4.2821 & 4.3043 & 2.0277 & 2.3626 & 2.7520 \\
\hline 0.02 & 4.2228 & 4.1610 & 4.1259 & 4.2640 & 4.2735 & 4.2960 & 1.9899 & 2.3414 & 2.7221 \\
\hline 0.04 & 4.1991 & 4.1353 & 4.0959 & 4.2520 & 4.2603 & 4.2804 & 1.9624 & 2.3034 & 2.7063 \\
\hline 0.06 & 4.1744 & 4.1141 & 4.0714 & 4.2395 & 4.2494 & 4.2676 & 1.9313 & 2.2622 & 2.6517 \\
\hline 0.08 & 4.1498 & 4.0908 & 4.0472 & 4.2270 & 4.2373 & 4.2549 & 1.9201 & 2.2511 & 2.6056 \\
\hline 0.10 & 4.1276 & 4.0722 & 4.0298 & 4.2157 & 4.2276 & 4.2457 & 1.9011 & 2.2217 & 2.5362 \\
\hline \multicolumn{10}{|c|}{$\mathrm{ZnSO}_{4}$ in $7.5 \%$ Lactose-Water } \\
\hline 0.00 & 4.1780 & 4.1266 & 4.0978 & 4.2413 & 4.2558 & 4.2814 & 1.9243 & 2.2754 & 2.6342 \\
\hline 0.01 & 4.1552 & 4.1067 & 4.0790 & 4.2297 & 4.2455 & 4.2716 & 1.9106 & 2.2561 & 2.6294 \\
\hline 0.02 & 4.1360 & 4.0887 & 4.0629 & 4.2200 & 4.2362 & 4.2631 & 1.8796 & 2.2373 & 2.5804 \\
\hline 0.04 & 4.1107 & 4.0670 & 4.0318 & 4.2071 & 4.2250 & 4.2468 & 1.8520 & 2.2226 & 2.5107 \\
\hline 0.06 & 4.0850 & 4.0397 & 4.0081 & 4.1939 & 4.2107 & 4.2343 & 1.8317 & 2.1902 & 2.4922 \\
\hline 0.08 & 4.0602 & 4.0184 & 3.9817 & 4.1811 & 4.1996 & 4.2203 & 1.8225 & 2.1771 & 2.4773 \\
\hline 0.10 & 4.0335 & 3.9933 & 3.9602 & 4.1673 & 4.1865 & 4.2089 & 1.7841 & 2.1452 & 2.4607 \\
\hline \multicolumn{10}{|c|}{$\mathrm{ZnSO}_{4}$ in $10 \%$ Lactose-Water } \\
\hline 0.00 & 4.0894 & 4.0572 & 4.0097 & 4.1961 & 4.2198 & 4.2351 & 1.7936 & 2.1318 & 2.5019 \\
\hline 0.01 & 4.0695 & 4.0372 & 3.9921 & 4.1859 & 4.2094 & 4.2258 & 1.7812 & 2.1160 & 2.4793 \\
\hline 0.02 & 4.0540 & 4.0208 & 3.9767 & 4.1779 & 4.2009 & 4.2177 & 1.7696 & 2.0987 & 2.4564 \\
\hline 0.04 & 4.0308 & 3.9882 & 3.9474 & 4.1660 & 4.1838 & 4.2021 & 1.7480 & 2.0700 & 2.4184 \\
\hline 0.06 & 4.0095 & 3.9670 & 3.9270 & 4.1549 & 4.1727 & 4.1912 & 1.7293 & 2.0541 & 2.4057 \\
\hline 0.08 & 3.9860 & 3.9434 & 3.9056 & 4.1427 & 4.1603 & 4.1798 & 1.7063 & 2.0340 & 2.3666 \\
\hline 0.10 & 3.9609 & 3.9271 & 3.8809 & 4.1297 & 4.1516 & 4.1665 & 1.6856 & 2.0181 & 2.3293 \\
\hline
\end{tabular}


Table 3: Calculated values of Internal pressure, $\pi_{\mathrm{i}}$, viscous relaxation time, $\tau$, relative association, $\mathrm{R}_{\mathrm{A}}$ of $\mathrm{ZnSO}_{4}$ in water, $2.5 \%$, $5.0 \%, 7.5 \%$ and $10 \%$ lactose-water mixed solvent at $303.15,308.15$ and $313.15 \mathrm{~K}$ temperature

\begin{tabular}{|c|c|c|c|c|c|c|c|c|c|}
\hline \multirow[b]{2}{*}{$\mathrm{C}$ mol. $\mathrm{kg}^{-1}$} & \multicolumn{3}{|c|}{$\pi_{\mathrm{I}} \times 10^{9} \mathrm{~Pa}$} & \multicolumn{3}{|c|}{$\tau \times 10^{13} \mathrm{~s}$} & \multicolumn{3}{|c|}{$\mathrm{R}_{\mathrm{A}}$} \\
\hline & $303.15 \mathrm{~K}$ & $308.15 \mathrm{~K}$ & $313.15 \mathrm{~K}$ & $303.15 \mathrm{~K}$ & $308.15 \mathrm{~K}$ & $313.15 \mathrm{~K}$ & $303.15 \mathrm{~K}$ & $308.15 \mathrm{~K}$ & $313.15 \mathrm{~K}$ \\
\hline \multicolumn{10}{|c|}{$\mathrm{ZnSO}_{4}$ in Water } \\
\hline 0.00 & 2.5955 & 2.5010 & 2.4208 & 4.7065 & 4.2118 & 3.8117 & & & \\
\hline 0.01 & 2.6027 & 2.5094 & 2.4250 & 4.7315 & 4.2434 & 3.8252 & 1.0019 & 1.0018 & 1.0016 \\
\hline 0.02 & 2.6029 & 2.5182 & 2.4264 & 4.7351 & 4.2742 & 3.8320 & 1.0037 & 1.0037 & 1.0027 \\
\hline 0.04 & 2.6053 & 2.5187 & 2.4290 & 4.7547 & 4.2916 & 3.8466 & 1.0067 & 1.0059 & 1.0049 \\
\hline 0.06 & 2.6073 & 2.5163 & 2.4320 & 4.7805 & 4.3081 & 3.8692 & 1.0090 & 1.0073 & 1.0074 \\
\hline 0.08 & 2.6128 & 2.5178 & 2.4303 & 4.8166 & 4.3321 & 3.8881 & 1.0119 & 1.0090 & 1.0091 \\
\hline 0.10 & 2.6131 & 2.5173 & 2.4314 & 4.8345 & 4.3485 & 3.9095 & 1.0141 & 1.0109 & 1.0114 \\
\hline \multicolumn{10}{|c|}{$\mathrm{ZnSO}_{4}$ in $2.5 \%$ Lactose-Water } \\
\hline 0.00 & 2.6121 & 2.5158 & 2.4290 & 4.8999 & 4.3887 & 3.9512 & & & \\
\hline 0.01 & 2.6169 & 2.5218 & 2.4356 & 4.9202 & 4.4113 & 3.9746 & 1.0014 & 1.0016 & 1.0016 \\
\hline 0.02 & 2.6207 & 2.5269 & 2.4409 & 4.9419 & 4.4323 & 3.9991 & 1.0028 & 1.0030 & 1.0027 \\
\hline 0.04 & 2.6326 & 2.5279 & 2.4440 & 5.0075 & 4.4487 & 4.0266 & 1.0058 & 1.0055 & 1.0055 \\
\hline 0.06 & 2.6355 & 2.5311 & 2.4468 & 5.0386 & 4.4766 & 4.0546 & 1.0087 & 1.0085 & 1.0075 \\
\hline 0.08 & 2.6363 & 2.5331 & 2.4488 & 5.0646 & 4.5005 & 4.0815 & 1.0112 & 1.0114 & 1.0101 \\
\hline 0.10 & 2.6462 & 2.5361 & 2.4521 & 5.1231 & 4.5299 & 4.1099 & 1.0138 & 1.0139 & 1.0125 \\
\hline \multicolumn{10}{|c|}{$\mathrm{ZnSO}_{4}$ in $5 \%$ Lactose-Water } \\
\hline 0.00 & 2.6253 & 2.5244 & 2.4436 & 5.1033 & 4.5408 & 4.1169 & & & \\
\hline 0.01 & 2.6303 & 2.5395 & 2.4495 & 5.1242 & 4.5972 & 4.1390 & 1.0017 & 1.0017 & 1.0016 \\
\hline 0.02 & 2.6456 & 2.5454 & 2.4569 & 5.1890 & 4.6243 & 4.1688 & 1.0034 & 1.0030 & 1.0030 \\
\hline 0.04 & 2.6539 & 2.5558 & 2.4587 & 5.2427 & 4.6779 & 4.1849 & 1.0061 & 1.0059 & 1.0059 \\
\hline 0.06 & 2.6646 & 2.5676 & 2.4722 & 5.3026 & 4.7400 & 4.2451 & 1.0092 & 1.0089 & 1.0090 \\
\hline 0.08 & 2.6648 & 2.5674 & 2.4829 & 5.3295 & 4.7612 & 4.2986 & 1.0113 & 1.0113 & 1.0116 \\
\hline 0.10 & 2.6682 & 2.5720 & 2.4988 & 5.3731 & 4.8144 & 4.3877 & 1.0130 & 1.0122 & 1.0126 \\
\hline \multicolumn{10}{|c|}{$\mathrm{ZnSO}_{4}$ in $7.5 \%$ Lactose-Water } \\
\hline 0.00 & 2.6499 & 2.5437 & 2.4582 & 5.3662 & 4.7748 & 4.3207 & & & \\
\hline 0.01 & 2.6556 & 2.5502 & 2.4590 & 5.3851 & 4.7977 & 4.3227 & 1.0018 & 1.0018 & 1.0019 \\
\hline 0.02 & 2.6692 & 2.5562 & 2.4736 & 5.4403 & 4.8219 & 4.3755 & 1.0036 & 1.0035 & 1.0037 \\
\hline 0.04 & 2.6796 & 2.5594 & 2.4942 & 5.4963 & 4.8468 & 4.4538 & 1.0070 & 1.0072 & 1.0072 \\
\hline 0.06 & 2.6850 & 2.5692 & 2.4976 & 5.5414 & 4.8950 & 4.4784 & 1.0093 & 1.0105 & 1.0106 \\
\hline 0.08 & 2.6854 & 2.5697 & 2.4983 & 5.5647 & 4.9217 & 4.4993 & 1.0119 & 1.0128 & 1.0129 \\
\hline 0.10 & 2.7004 & 2.5768 & 2.4989 & 5.6469 & 4.9762 & 4.5263 & 1.0143 & 1.0142 & 1.0147 \\
\hline \multicolumn{10}{|c|}{$\mathrm{ZnSO}_{4}$ in $10 \%$ Lactose-Water } \\
\hline 0.00 & 2.6845 & 2.5731 & 2.4774 & 5.6767 & 5.0439 & 4.5091 & & & \\
\hline 0.01 & 2.6911 & 2.5788 & 2.4842 & 5.6957 & 5.0642 & 4.5334 & 1.0025 & 1.0018 & 1.0019 \\
\hline 0.02 & 2.6945 & 2.5850 & 2.4907 & 5.7215 & 5.0898 & 4.5605 & 1.0034 & 1.0036 & 1.0035 \\
\hline 0.04 & 2.7017 & 2.5941 & 2.5008 & 5.7737 & 5.1336 & 4.6074 & 1.0063 & 1.0066 & 1.0065 \\
\hline 0.06 & 2.7070 & 2.5956 & 2.5009 & 5.8228 & 5.1682 & 4.6302 & 1.0089 & 1.0087 & 1.0091 \\
\hline 0.08 & 2.7150 & 2.6004 & 2.5109 & 5.8802 & 5.2064 & 4.6862 & 1.0116 & 1.0115 & 1.0119 \\
\hline 0.10 & 2.7211 & 2.6015 & 2.5185 & 5.9334 & 5.2455 & 4.7419 & 1.0136 & 1.0133 & 1.0132 \\
\hline
\end{tabular}

The apparent molal volume, $\phi_{v}$ shows positive value and increasing trends with lactose content in the solution (Fig. 6 and 7). Similarly, the limiting apparent molal volume, $\phi_{v}^{0}$ provides information about ion-solvent interactions, which shows positive variation. Positive variations for both $\phi_{v}$ and $\phi_{v}^{0}$ (Table 4 and 5) are indicative of strong solute-solvent interactions in the systems (Parmar and Thakur, 2006; Rathore and Singh, 2006; Ali et al., 2002). Pinto et al. (2015) and Naseem and Ashraf (2016) have also reported similar type of observation for their systems. The constant $S_{v}$, is indicative of ion-ion interaction and the values are positive but smaller in comparison to $\phi_{v}^{0}$.
According to Ali and Shahjahan (2016) such type of variations are due to weak ion-ion interactions.

The parameter, internal pressure is the cohesive force, which is due to the attractive and repulsive forces between the molecules (Kannappan et al., 2009). In the present systems, there is increase in internal pressure with the concentration of both $\mathrm{ZnSO}_{4}$ as well as lactose (Fig 8). This can be explained due to the strengthening of cohesive forces obtained during the structure making of solvents in presence of solute (Suryanarayan and Kuppu Samy, 1981). This force, however decreases with the increase in temperature because of dispersion of solute molecules (Table. 3). 
Table 4: Calculated values of apparent molal volume, $\phi_{\mathrm{v}}$, apparent molal compresssibility, $\phi_{\mathrm{K}}$, solvation number, $\mathrm{S}_{\mathrm{n}}$ of $\mathrm{ZnSO}_{4}$ in water, $2.5 \%, 5.0 \%, 7.5 \%$ and $10 \%$ lactose-water mixed solvent at $303.15,308.15$ and $313.15 \mathrm{~K}$ temperature

\begin{tabular}{|c|c|c|c|c|c|c|c|c|c|}
\hline \multirow[b]{2}{*}{$\mathrm{C}$ mol.kg ${ }^{-1}$} & \multicolumn{3}{|c|}{$\phi_{\mathrm{v}} \times 10^{3} \mathrm{dm}^{3} \cdot \mathrm{mol}^{-1}$} & \multicolumn{3}{|c|}{$-\phi \mathrm{K} \times 10^{10} \mathrm{~N}^{-1} \cdot \mathrm{dm}^{5} \cdot \mathrm{mol}^{-1}$} & \multicolumn{3}{|c|}{$\mathrm{S}_{\mathrm{n}}$} \\
\hline & $303.15 \mathrm{~K}$ & $308.15 \mathrm{~K}$ & $313.15 \mathrm{~K}$ & $303.15 \mathrm{~K}$ & $308.15 \mathrm{~K}$ & $313.15 \mathrm{~K}$ & $303.15 \mathrm{~K}$ & $308.15 \mathrm{~K}$ & $313.15 \mathrm{~K}$ \\
\hline \multicolumn{10}{|c|}{$\mathrm{ZnSO}_{4}$ in Water } \\
\hline 0.00 & & & & & & & & & \\
\hline 0.01 & 56.78 & 56.53 & 66.41 & 1.771 & 2.032 & 2.375 & 25.1256 & 23.7347 & 29.3004 \\
\hline 0.02 & 66.69 & 61.44 & 91.52 & 1.561 & 1.756 & 2.042 & 23.0942 & 22.9536 & 28.6195 \\
\hline 0.04 & 86.43 & 101.36 & 106.30 & 1.366 & 1.361 & 1.820 & 21.6402 & 21.2529 & 27.5655 \\
\hline 0.06 & 104.45 & 127.83 & 114.32 & 1.197 & 1.094 & 1.538 & 20.4054 & 19.6480 & 24.6731 \\
\hline 0.08 & 106.99 & 135.83 & 129.49 & 1.109 & 1.046 & 1.218 & 19.3730 & 19.5751 & 21.5296 \\
\hline 0.10 & 113.37 & 139.46 & 133.38 & 1.075 & 1.003 & 1.091 & 19.3124 & 19.3362 & 20.2581 \\
\hline \multicolumn{10}{|c|}{$\mathrm{ZnSO}_{4}$ in $2.5 \%$ Lactose-Water } \\
\hline 0.00 & & & & & & & & & \\
\hline 0.01 & 97.90 & 87.87 & 87.70 & 1.578 & 1.585 & 1.397 & 25.2809 & 24.9889 & 22.6716 \\
\hline 0.02 & 107.58 & 92.64 & 112.33 & 1.265 & 1.486 & 1.190 & 21.8511 & 23.9419 & 21.3532 \\
\hline 0.04 & 114.59 & 109.57 & 121.86 & 0.903 & 1.294 & 0.874 & 17.5687 & 22.3002 & 17.7248 \\
\hline 0.06 & 116.66 & 111.65 & 131.38 & 0.811 & 1.082 & 0.822 & 16.4294 & 19.6393 & 17.4554 \\
\hline 0.08 & 122.40 & 113.73 & 133.46 & 0.758 & 0.987 & 0.723 & 15.9940 & 18.4660 & 16.2233 \\
\hline 0.10 & 123.75 & 117.78 & 134.55 & 0.726 & 0.910 & 0.701 & 15.6774 & 17.7222 & 15.9961 \\
\hline \multicolumn{10}{|c|}{$\mathrm{ZnSO}_{4}$ in $5 \%$ Lactose-Water } \\
\hline 0.00 & & & & & & & & & \\
\hline 0.01 & 79.30 & 79.20 & 88.75 & 1.515 & 1.377 & 1.394 & 23.7169 & 22.1231 & 22.9116 \\
\hline 0.02 & 83.97 & 98.43 & 98.28 & 1.281 & 1.269 & 1.260 & 20.9153 & 21.7693 & 21.6570 \\
\hline 0.04 & 105.39 & 107.76 & 102.77 & 0.958 & 1.027 & 1.151 & 17.8755 & 19.0415 & 20.4225 \\
\hline 0.06 & 107.43 & 110.61 & 105.64 & 0.891 & 0.875 & 1.022 & 17.0482 & 17.1507 & 18.8050 \\
\hline 0.08 & 117.92 & 117.89 & 110.52 & 0.818 & 0.802 & 0.940 & 16.5900 & 16.5159 & 17.9279 \\
\hline 0.10 & 127.93 & 135.67 & 129.81 & 0.736 & 0.659 & 0.758 & 16.0750 & 15.5836 & 16.5781 \\
\hline \multicolumn{10}{|c|}{$\mathrm{ZnSO}_{4}$ in $7.5 \%$ Lactose-Water } \\
\hline 0.00 & & & & & & & & & \\
\hline 0.01 & 51.76 & 60.97 & 60.64 & 2.013 & 1.701 & 1.582 & 28.8530 & 25.4406 & 24.0218 \\
\hline 0.02 & 61.15 & 70.38 & 65.30 & 1.796 & 1.566 & 1.439 & 26.5470 & 24.1978 & 22.3282 \\
\hline 0.04 & 77.47 & 79.61 & 72.19 & 1.323 & 1.133 & 1.325 & 21.2278 & 18.9912 & 21.1066 \\
\hline 0.06 & 96.90 & 84.04 & 80.63 & 1.118 & 1.078 & 1.140 & 19.4972 & 18.4219 & 19.0898 \\
\hline 0.08 & 104.05 & 99.15 & 94.21 & 1.015 & 0.925 & 1.047 & 18.4746 & 17.1513 & 18.4840 \\
\hline 0.10 & 109.13 & 113.78 & 107.94 & 0.971 & 0.849 & 0.920 & 18.1764 & 16.9314 & 17.5600 \\
\hline \multicolumn{10}{|c|}{$\mathrm{ZnSO}_{4}$ in $10 \%$ Lactose-Water } \\
\hline 0.00 & & & & & & & & & \\
\hline 0.01 & 64.92 & 62.39 & 62.25 & 1.900 & 1.680 & 1.462 & 25.3409 & 25.4828 & 22.7820 \\
\hline 0.02 & 76.51 & 66.94 & 76.21 & 1.403 & 1.490 & 1.299 & 22.4961 & 23.2169 & 21.3307 \\
\hline 0.04 & 97.20 & 80.68 & 87.62 & 1.026 & 1.348 & 1.165 & 18.5729 & 21.9901 & 20.0928 \\
\hline 0.06 & 108.51 & 105.30 & 103.68 & 0.854 & 1.038 & 0.930 & 16.8327 & 19.1039 & 17.7268 \\
\hline 0.08 & 111.64 & 108.05 & 108.00 & 0.806 & 0.951 & 0.841 & 16.2965 & 18.0373 & 16.6933 \\
\hline 0.10 & 118.97 & 120.78 & 121.69 & 0.773 & 0.789 & 0.778 & 16.2361 & 16.5242 & 16.5473 \\
\hline
\end{tabular}

Table 5: Acoustic Pararmeters of $\mathrm{ZnSO}_{4}$ in water and different proportions of lactose water mixture at 303.15, 308.15 and 313.15K temperature

\begin{tabular}{|c|c|c|c|c|c|c|c|c|c|c|c|c|}
\hline \multirow[b]{2}{*}{ Solvent } & \multicolumn{3}{|c|}{$\phi_{v}^{0} \times 10^{3}\left(\mathrm{dm}^{3} \cdot \mathrm{mol}^{-1}\right)$} & \multicolumn{3}{|c|}{$-\phi_{k}^{0} \times 10^{9}\left(\mathrm{~N}^{-1} \cdot \mathrm{dm}^{5} \cdot \mathrm{mol}^{-1}\right)$} & \multicolumn{3}{|c|}{$\mathrm{S}_{\mathrm{v}}$} & \multicolumn{3}{|c|}{$\mathrm{S}_{\mathrm{k}} \times 10^{9}\left(\mathrm{~N}^{-1} \cdot \mathrm{dm}^{13 / 2} \cdot \mathrm{mol}^{-3 / 2}\right)$} \\
\hline & $303.15 \mathrm{~K}$ & $308.15 \mathrm{~K}$ & $313.15 \mathrm{~K}$ & $303.15 \mathrm{~K}$ & $308.15 \mathrm{~K}$ & $313.15 \mathrm{~K}$ & $303.15 \mathrm{~K}$ & $308.15 \mathrm{~K}$ & $313.15 \mathrm{~K}$ & $303.15 \mathrm{~K}$ & $308.15 \mathrm{~K}$ & $313.15 \mathrm{~K}$ \\
\hline $\mathrm{ZnSO}_{4}$ in Water & 275.5 & 436.0 & 295.6 & 3.264 & 4.962 & 5.891 & 30.08 & 10.31 & 43.55 & 2.045 & 2.445 & 2.943 \\
\hline 2.5\% Lactose-Water & 114.1 & 141.5 & 201.6 & 3.880 & 3.293 & 3.246 & 89.35 & 75.21 & 77.01 & 1.838 & 1.929 & 1.646 \\
\hline 5\% Lactose-Water & 224.2 & 218.7 & 153.3 & 3.509 & 3.33 & 2.722 & 55.60 & 61.39 & 73.11 & 1.785 & 1.714 & 1.670 \\
\hline 7.5\% Lactose-Water & 282.4 & 224.3 & 209.1 & 5.102 & 4.083 & 2.997 & 22.89 & 36.59 & 35.34 & 2.465 & 2.083 & 1.884 \\
\hline $10 \%$ Lactose-Water & 252.9 & 283.5 & 262.8 & 5.012 & 4.104 & 3.253 & 42.10 & 29.93 & 36.93 & 2.200 & 2.095 & 1.776 \\
\hline
\end{tabular}

In the present study, the relaxation time, $\tau$, is in the order of $10^{-13}$ and according to Kinsler and Rray (1989) this is due to structural relaxation process, which again suggests these molecules to be rearranged due to supportive process (Sawhney et al., 2014) (Fig. 9). The relative association, $R_{A}$ is the parameter that depends on the structure breaking behaviour of solute when is added to solvent and the solvation of ions by the free solvent molecules (Palani and Geeta, 2007). However, the increase in $R_{A}$ is associated with the later effect as per the literature (Ali et al., 2001). Therefore in the present investigation, the solvation of solute predominates over the breaking of the solvent 
aggregates. Jahagirdar and Shankarwar (2000) have also reported similar kind of observations.

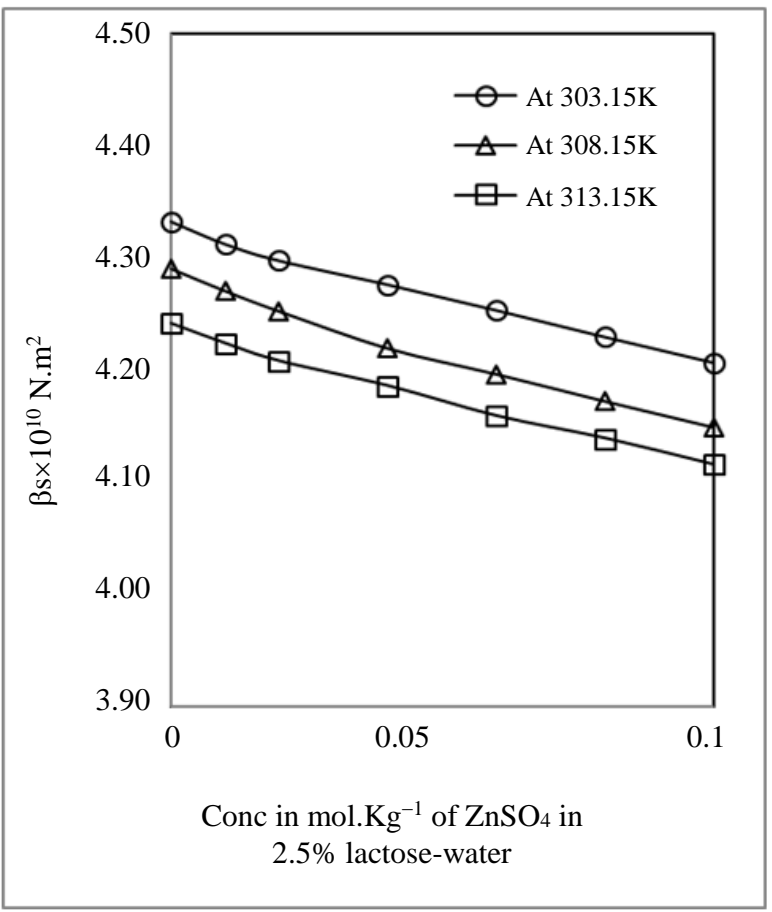

Fig. 2: Variation of isentropic compressibility, $\beta_{s}$ of $\mathrm{ZnSO}_{4}$ in $2.5 \%$ Lactose-water $v s$. temperature

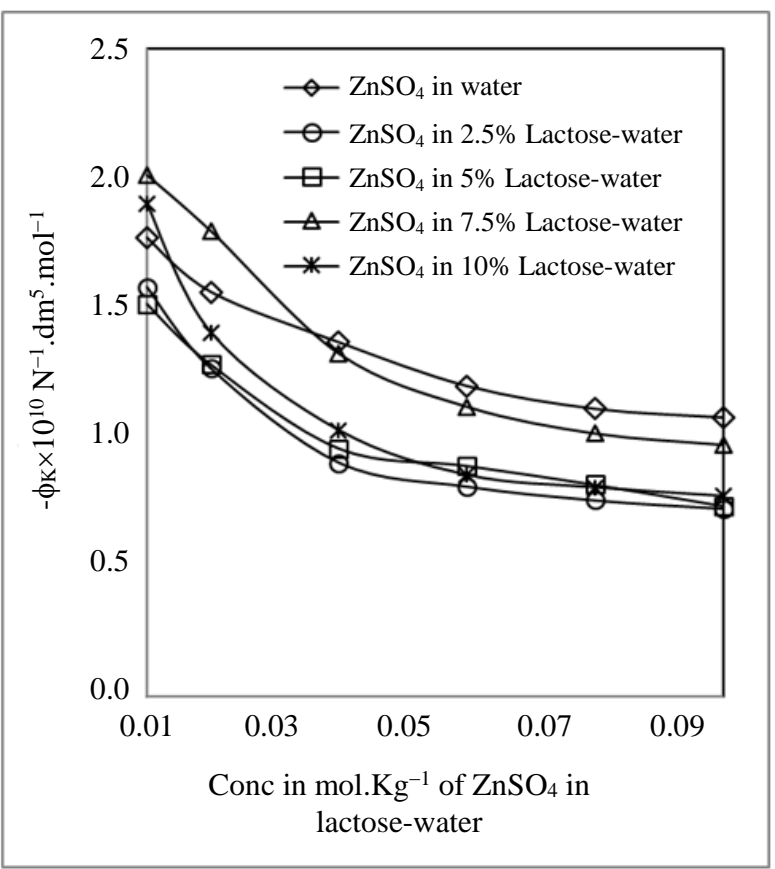

Fig. 3: Variation of apparent molal compressibility, $\phi_{k}$ vs. Concentration of $\mathrm{ZnSO}_{4}$ in water and Lactose-water mixed solvent at $303.15 \mathrm{~K}$

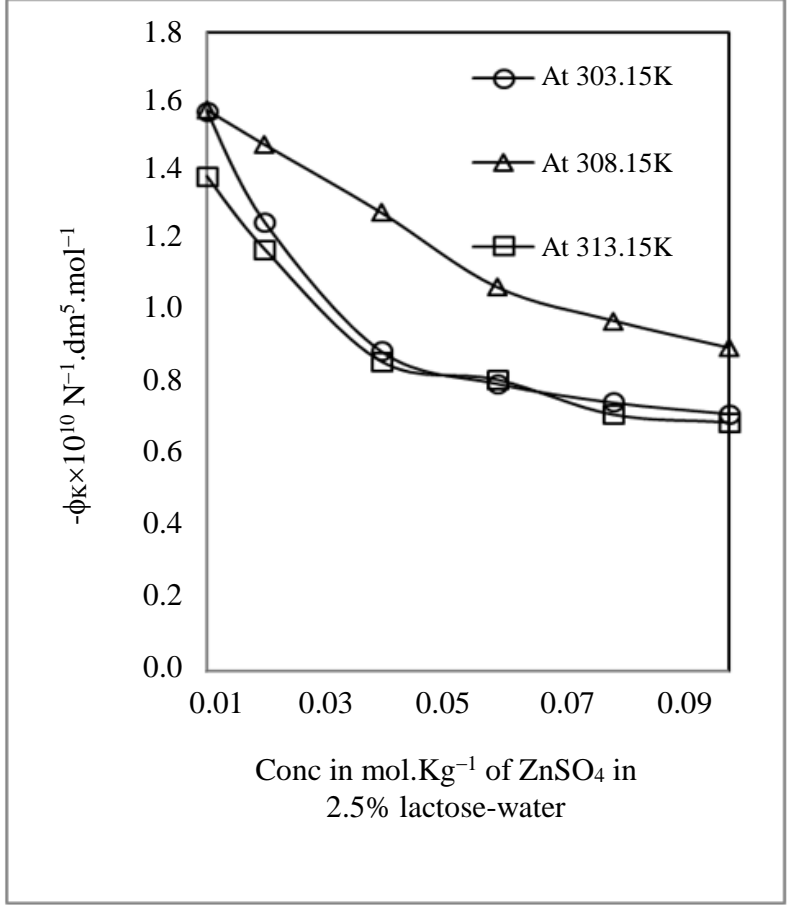

Fig. 4: Variation of apparent molal compressibility, $\phi_{k}$ of $\mathrm{ZnSO}_{4}$ in $2.5 \%$ Lactose-water $v s$. Temperature

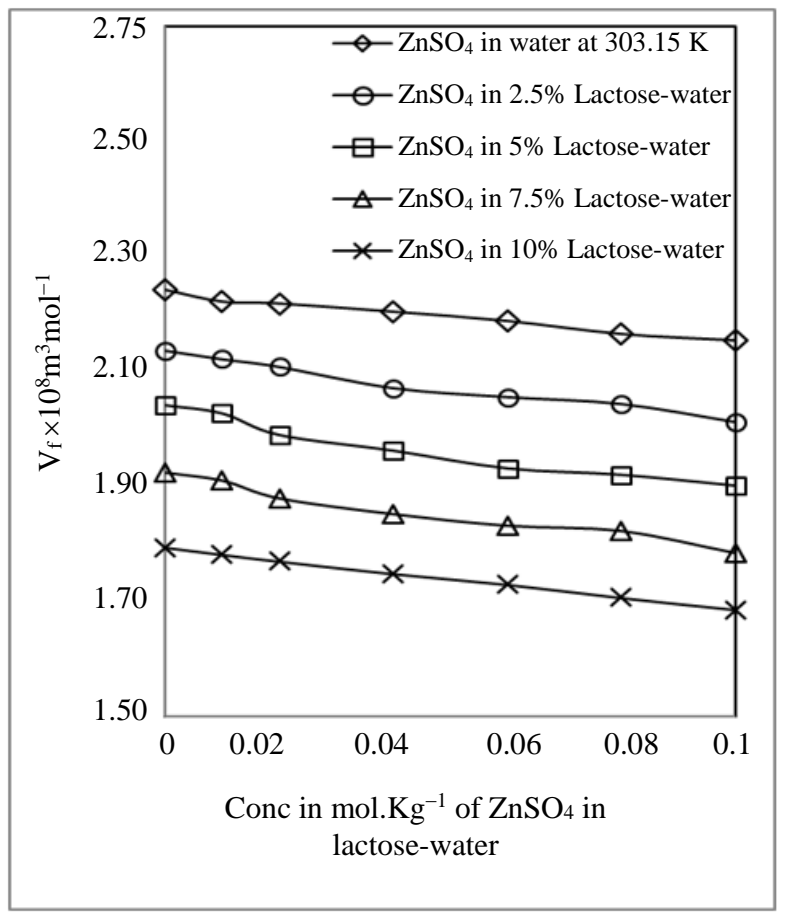

Fig. 5: Variation of free volume, $V_{f} v s$. Concentration of $\mathrm{ZnSO}_{4}$ in water and Lactose-water mixed solvent at $303.15 \mathrm{~K}$ 


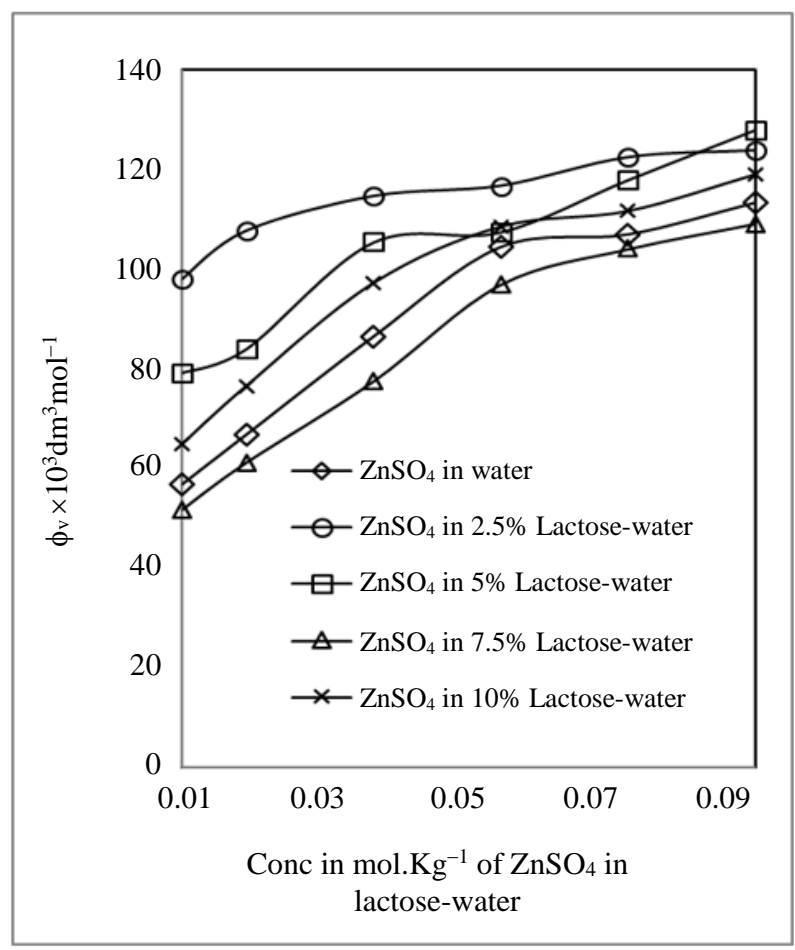

Fig. 6: Variation of apparent molal volume, $\phi_{v}$ vs. Concentration of $\mathrm{ZnSO}_{4}$ in water and Lactose-water mixed solvent at $303.15 \mathrm{~K}$

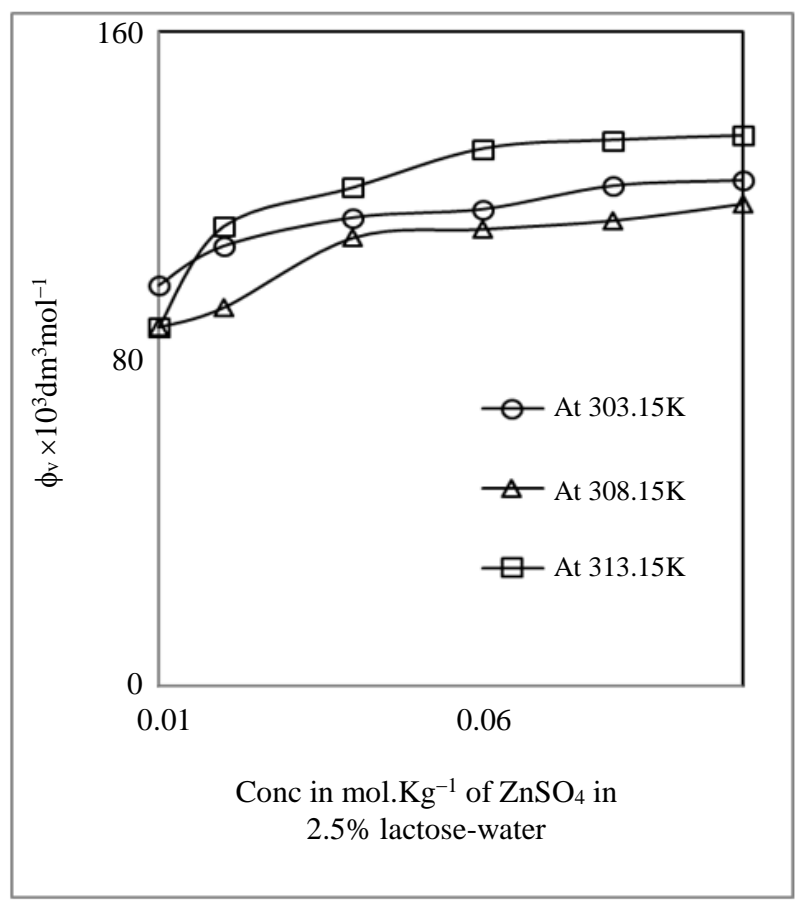

Fig. 7: Variation of apparent molal volume, $\phi_{v}$ of $\mathrm{ZnSO}_{4}$ in 2.5\% Lactose-water vs temperature

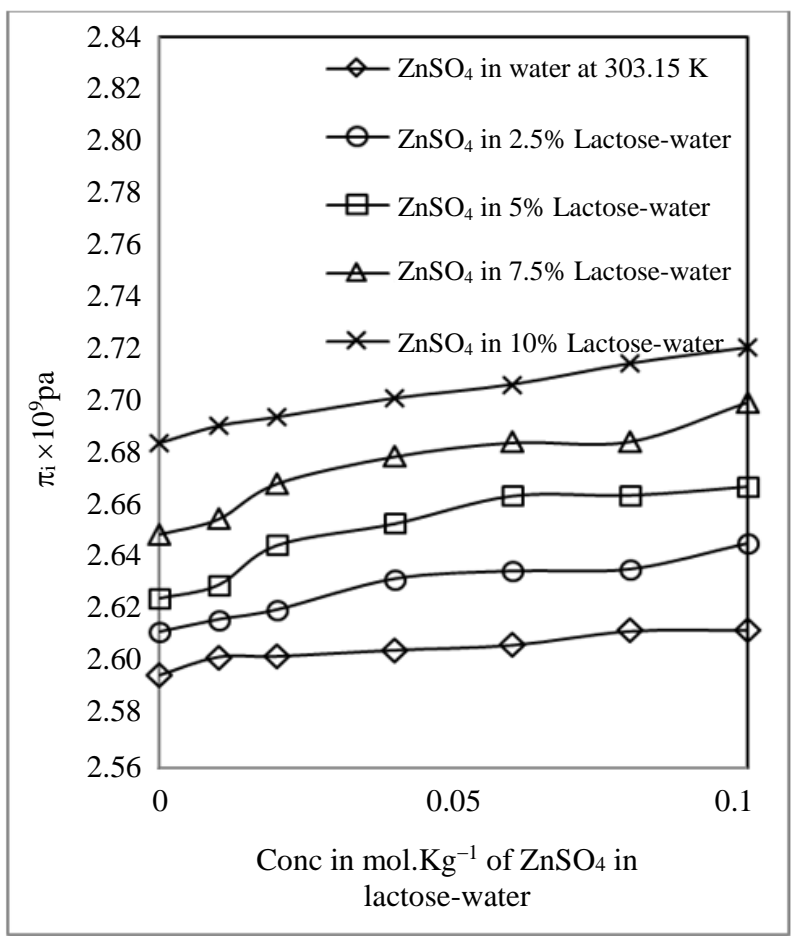

Fig. 8: Variation of internal pressure, $\pi_{\mathrm{i}} v s$. Concentration of $\mathrm{ZnSO}_{4}$ in water and Lactose-water mixed solvent at $303.15 \mathrm{~K}$

Solvation number explains the interaction of solute with the solvent molecules present in the solutions. The positive values of solvation number in the present study indicate appreciable solvation of solutes (Mehraand and Sajnami, 2000). This is indicative of structure-promoting nature of soulte and the positive values also suggest the compressibility to be less in solution in comparison to solvent. This increases the ion mobility and so also the probability to interact with the solvent molecules. The solvent molecules are attached to the ions by strong covalent bonds in the primary sheath of solvation and in the secondary sheath, there are weak forces of attraction between solute and solvent species (Dash and Kamila, 2017). In the present investigation, the solvation number decreases as the concentration of solute increases (Fig. 10). This is attributed to the decrease in the size of the secondary sheath of solvation. More over, $S_{n}$ decreases with increase in temperature, which shows weakening of solute-solvent interaction (Fig. 11). According to Marcus (2005), this number depends on both the ion and solvent involved as well as on the concentration through the interactions of this ion with other ions. However, the non-linear variation of solvation number with $\mathrm{ZnSO}_{4}$, indicates the increase in ion-solvent interactions (Palani et al., 2001). 


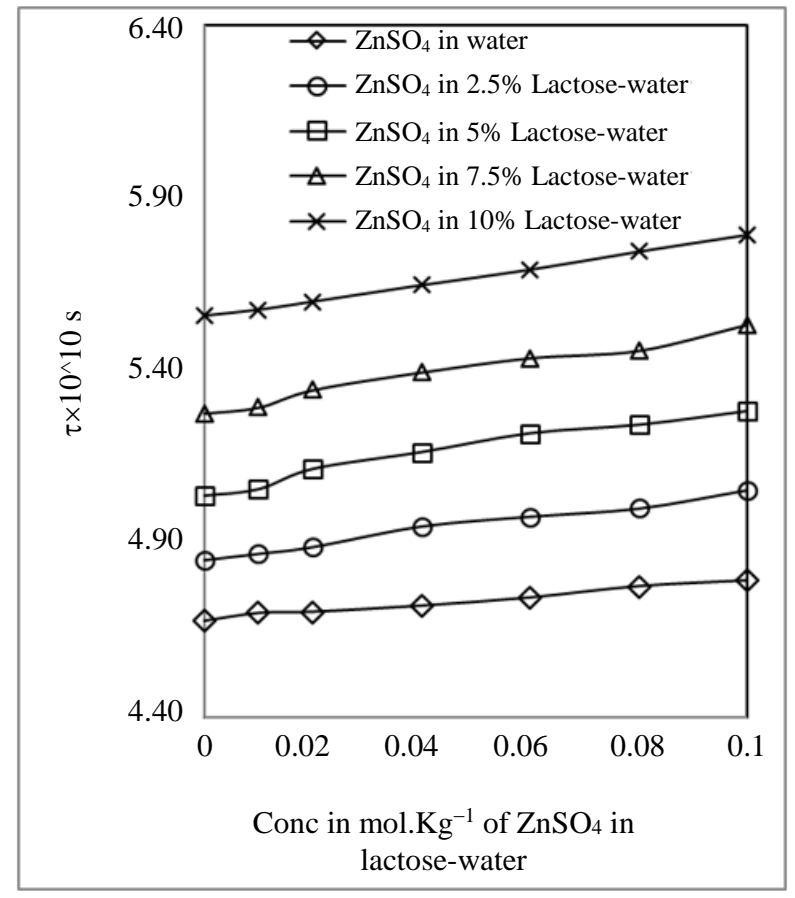

Fig. 9: Variation of viscus relaxation time, $\tau$ vs. Concentration of $\mathrm{ZnSO}_{4}$ in water and Lactose-water mixed solvent at $303.15 \mathrm{~K}$

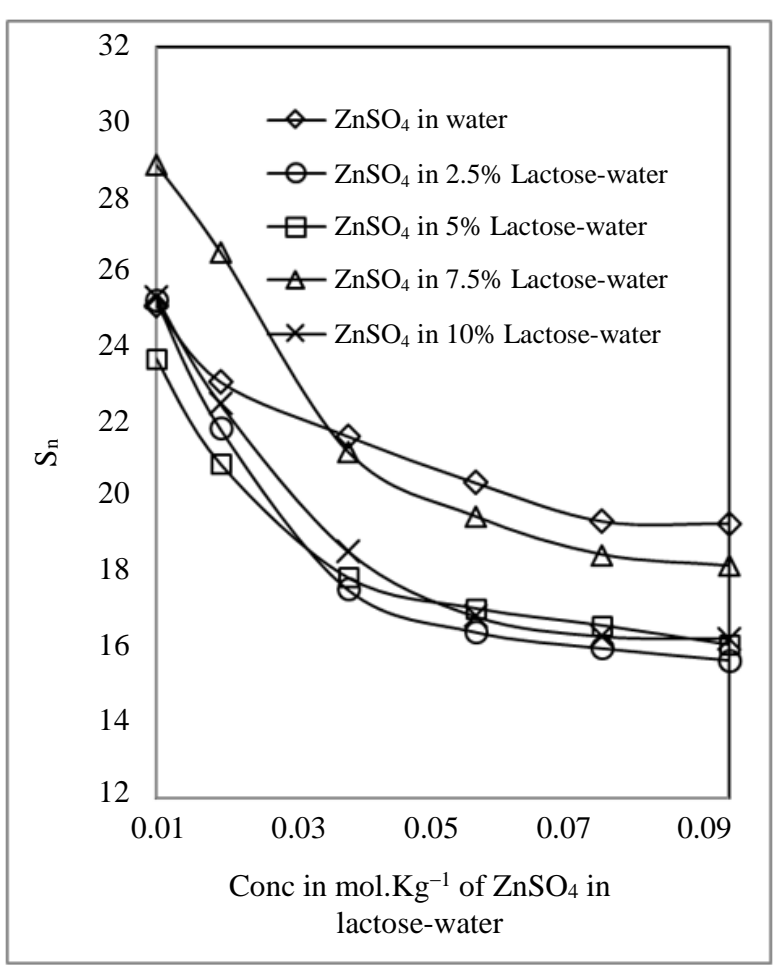

Fig. 10: Variation of Solvation number, $S_{\mathrm{n}} v s$. Concentration of $\mathrm{ZnSO}_{4}$ in water and Lactose-water mixed solvent at $303.15 \mathrm{~K}$

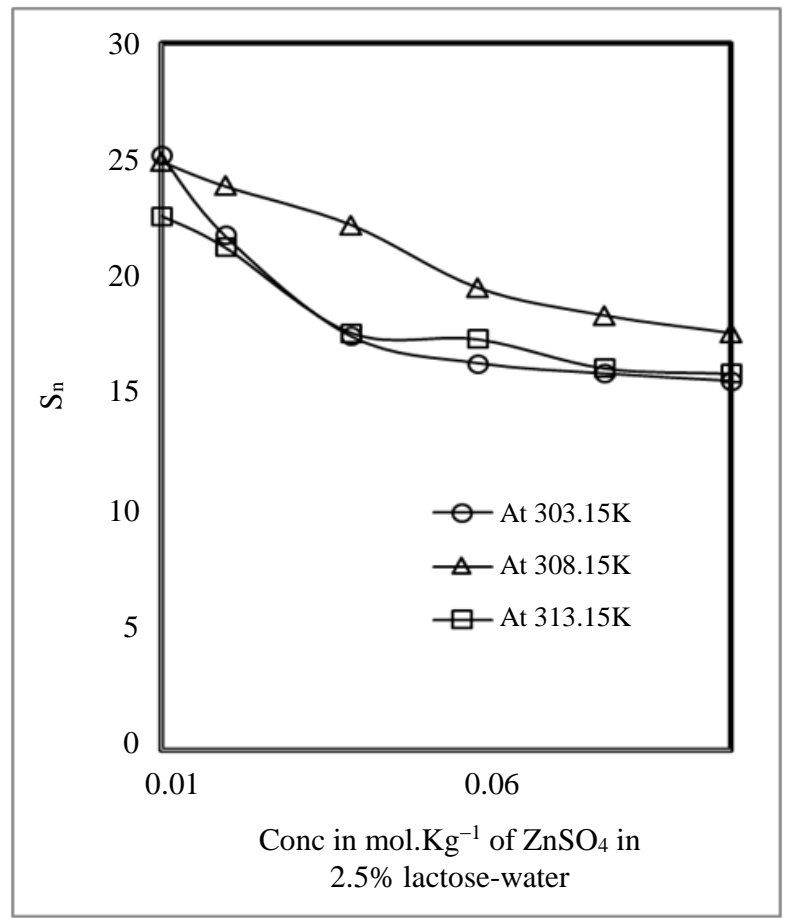

Fig. 11: Variation of Solvation number, $\mathrm{S}_{\mathrm{n}}$ of $\mathrm{ZnSO}_{4}$ in $2.5 \%$ Lactose-water $v s$. temperature

\section{Conclusion}

Acoustic studies involving ultrasonic velocity measurement are non-destructive techniques to access the molecular association among the components through ion-solvation. Besides, different other supporting parameters such as isentropic compressibility, internal pressure and apparent molal volume etc., have been evaluated at three different temperatures. The present study reports the existence of solute-solvent and solute-solute interaction between $\mathrm{ZnSO}_{4}$ and aqueous lactose solution. From the Results and discussion, it reveals the structure-promoting tendency of added solute. The electrostrictive solvation of ions is supported by partial molal volume. The breaking of solvent structure was also confirmed from internal pressure as well as relative association, where the solvation of solute predominates over the breaking of the solvent aggregates. $S_{V}$ and $S_{K}$ values show weak ion-ion interaction in the systems. The solvation number indicates appreciable ion solvation along with structure promoting nature of solute. However, the ion solvation decreases as the temperature increases. So overall, it can be concluded that the strength of interaction increases with increase in lactose as well as $\mathrm{ZnSO}_{4}$ concentration. The decrease in solvation number with the increase in lactose concentration is indicative of the dehydrating effect of co-solute on $\mathrm{ZnSO}_{4}$. This result would be of great importance to pharmaceutical industries for the 
drugs related to lactose and $\mathrm{ZnSO}_{4}$ for designing and delivery systems.

\section{Acknowledgement}

One of the authors (GDN) is thankful to Visvesvaraya Technological University (VTU), Belagavi, Karnataka for giving the opportunity to work for his Ph.D. programme.

\section{Author's Contributions}

Ganesh Durgachalam Natraj: Ganesh is the first author and did all the experimental work along with data collection.

Vinay Sathyanarayan Bhat: Vinay's contributions involve the evaluation and calculation part of different parametrs.

Susmita Kamila: Susmita is the supervisor and the corresponding author. Her contribution includes Research planning, organizational designing and framework of the manuscript.

\section{Conflict of Interest}

The authors declares that they have no conflict of interest.

\section{References}

Ali, A. and Shahjahan, 2016. Volumetric, viscometric and refractive index behavior of some $\alpha$-amino acids in aqueous tetrapropylammonium bromide at different temperatures. J. Iranian Chem. Soc., 3: 340-350. DOI: 10.1007/BF03245957

Ali, A., A.K. Nain, N. Kumar and M. Ibrahim, 2002. Density and viscosity of magnesium sulphate in formamide + ethylene glycol mixed solvents. J. Chem. Sci., 114: 495-500. DOI: $10.1007 / \mathrm{BF} 02704193$

Ali, A., Abida, A.K. Nain and S. Hyder, 2001. Study of intermolecular interaction in binary liquid mixtures through ultrasonic speed measurement. Ind. J. Pure. Applied Ultrason, 23: 73-79.

Broadley, M.R., P.J. White, J.P. Hammond, I. Zelko and A. Lux, 2007. Zinc in plants. New Phytol., 173: 677-702. DOI: 10.1111/j.1469-8137.2007.01996.x

Chauhan, S., P. Chaudhary, K. Sharma, K. Kumar and Kiran, 2013. Temperature-dependent volumetric and viscometric properties of amino acids in aqueous solutions of an antibiotic drug. Chem. Papers, 67: 1442-1452.

DOI: 10.2478/s11696-013-0404-y

Coleman, J.E., 1967. Metal ion dependent binding of sulphonamide to carbonic anhydrase. Nature, 214: 193-194. DOI: $10.1038 / 214193 \mathrm{a} 0$
Dash, J.K. and S. Kamila, 2015. Ion-solvent interactions in lanthanum(III) chloride and D-glucose-water mixed solvent systems: An ultrasonic study. Russian J. Physical Chem. A, 89: 1578-1584. DOI: $10.1134 /$ S0036024415090071

Dash, J.K. and S. Kamila, 2017. Ion solvation in salts of biologically important rare earth metals with aqueous sugar alcohol (sorbitol) mixed-solvent systems J. Taibah Univ. Sci., 11: 801-807.

DOI: $10.1016 /$ j.jtusci.2016.10.007

Debye, P. and C. Huckel, 1923. Z. Phy., 24: 305-305.

Dhanalakshmi, A. and E.J. Vasantharani, 1999. Analysis of apparaent molal volume and apparaent molal compressibility of quaternary ammonium salt in non- aqueous medium. J. Pure. Applied Ultrason, 21: 79-82.

Evans, G.W., 1986. Zinc and its deficiency diseases. Clin. Physiol. Biochem., 4: 94-98. PMID: 3514057

Fort, R.J. and W.R. Moore, 1965. Adiabatic compressibilities of binary liquid mixtures. Trans. Faraday Soc., 61: 2102-2111. DOI: $10.1039 /$ TF9656102102

Franks, F., M.J. Tait and A.A. Suggettaetal, 1972. Hydration of monosaccharides: A study by dielectric and nuclear magnetic relaxation. J. Solut. Chem., 1: 131-151. DOI: 10.1007/BF01028450

Gucker Jr., F.T., 1933. The compressibility of solutions. I. The apparent molal compressibility of strong electrolytes. J. Am. Chem. Soc., 55: 2709-2718. DOI: $10.1021 / \mathrm{ja} 01334 \mathrm{a} 013$

Gupta, M.N., 1991. Thermostabilization of proteins. Biotechnol. Applied Biochem., 14: 1-11.

Jahagirdar, D.V. and A.G. Shankarwar, 2000. Indian J. Pure Applied Ultrasoics, 38: 760-760.

Jones, G. and M. Dole, 1929. The viscosity of aqueous solutions of strong electrolytes with special reference to barium chloride. J. Am. Chem. Soc., 51: 2950-2964. DOI: 10.1021/ja01385a012

Kamila, S. and J.K. Dash, 2012. Studies on molecular interactions in different solvent extractants with nbutanol at temperature $303.15 \mathrm{~K}$ : A thermoacoustic investigation. J. Mol. Liq., 172: 71-75. DOI: 10.1016/j.molliq.2012.05.010

Kamila, S., D.N. Ganesh and J. K. Dash, 2017. Ion solvation in biologically active magnesium salt with lactose-water mixed solvent system at different temperatures: A thermo-acoustics study. Phys. Chem. Liquids, 55: 396-409. DOI: 10.1080/00319104.2016.1218492

Kamila, S., S. Jena and B.B. Swain, 2005. Studies on thermo-acoustic parameters in binary liquid mixtures of phosphinic acid (Cyanex 272) with different diluents at temperature $303.15 \mathrm{~K}$ : An ultrasonic study. J. Chem. Thermodyn., 37: 820-825. DOI: $10.1016 /$ j.jct.2004.12.001 
Kamila, S., V. Chakravortty and S. Jena, 2004. Studies on acoustic behavior, viscosity and density of some commercial extractants and their molecular interaction with diluent, modifier and extractant. J. Soln. Chem., 33: 365-380. DOI: 10.1023/B:JOSL.0000036307.76436.a0

Kannappan, A.N. and R. Palani, 2007. Acoustical behaviour of glycerine, dextrose and sucrose in $\mathrm{Na}_{2} \mathrm{CO}_{3}$ and $\mathrm{NaHCO}_{3}$ buffer solution. Indian $\mathrm{J}$. Pure Applied Phys., 45: 573-579.

Kannappan, A.N., S. Thirumaran and R. Palani, 2009. Volumetric and thermodynamic studies of molecular interactions in ternary liquid mixtures at 303, 308 and 313K. J. Phys. Sci., 20: 97-108.

Kannappan, V. and S.C. Vinayagam, 2006. Ultrasonic study of ion-solvent interactions in aqueous and non-aqueous solutions of transition and inner transition metal ions. Ind. J. Pure Applied Phys., 44: 670-676.

Kannappan, V. and S.C. Vinayagam, 2007. Ultrasonic investigation of ion-solvent interactions in aqueous and non-aqueous solutions of transition and inner transition metal ions. Ind. J. Pure Applied Phys., 45: $143-150$.

Katrinak, T., L. Hneedkovsky and I. Cibulka, 2012. Partial molar volumes and partial molar isentropic compressions of three polyhydric alcohols derived from propane at infinite dilution in water at temperatures $\mathrm{T}=(278$ to 318$) \mathrm{K}$ and atmospheric pressure. J. Chem. Eng. Data, 57: 1152-1159. DOI: $10.1021 /$ je201184b

Kaulgud, M.V., 1976. Zet Fur Physk Chem., 36: 277-277.

Khan, W.U. and D.W. Sellen, 2011. e-Library of Evidence for Nutrition Actions, University of Toronto, Toronto, Canada.

Kinsler, L.E. and A.R. Rray, 1989. Fundamental of acoustic. Wiley Eastern, New Delhi.

Lane, T.W. and F.M.M. Morel, 2006. A biological function for cadmium in marine diatoms. Proc. National Acad. Sci., 97: 4627-4631. DOI: $10.1073 /$ pnas.090091397.

Marcus, Y., 2005. Electrostriction, ion solvation and solvent release on ion pairing. J. Phys. Chem. B, 109: 18541-18549. DOI: 10.1021/jp051505k

Masson, D.O., 1929. Solute molecular volumes in relation to solvation and ionization. Philos. Magazine, 8: 218-235. DOI: 10.1080/14786440808564880

Mehraand, R. and H. Sajnami, 2000. Ind. J. Pure Applied Phys., 38: 762-762.

Nain, A.K., M. Lather and Neetu, 2013b. Probing solute-solute and solute-solvent interactions in $(l$ arginine $+d$-xylose $/ l$-arabinose + water $)$ solutions at different temperatures by using volumetric and viscometric methods. J. Chem. Thermodynam., 63: 67-73. DOI: 10.1016/j.jct.2013.04.001
Nain, A.K., R. Pal and Neetu, 2013a. Volumetric, ultrasonic and viscometric studies of solute-solute and solute-solvent interactions of 1-threonine in aqueous-sucrose solutions at different temperatures. J. Chem. Thermodynam., 64: 172-181. DOI: $10.1016 /$ j.jct.2013.05.012

Naseem, B. and N. Ashraf, 2016. Volumetric behavior of nitroimidazoles in binary solvent mixtures. J. Mol. Liq., 224: 377-386. DOI: $10.1016 /$ j.molliq.2016.10.004

Nikam, P.S., H.R. Ansari and M. Hasan, 2000. Acoustical properties of fructose and maltose solutions in water and in aqueous $0.5 \mathrm{M} \mathrm{NH}_{4} \mathrm{Cl}$. J. Mol. Liq., 84: 169-178.

DOI: $10.1016 / \mathrm{S} 0167-7322(99) 00177-4$

Nithiyanantham, S. and L. Palaniappan, 2013. Physicochemical studies on some disaccharides (sucrose, lactose, maltose) in aqueous media at 298.15 K. Chem. Sci. Trans., 2: 35-40.

DOI: $10.7598 /$ cst2013.262

Pal, A. and N. Chauhan, 2011. Partial molar adiabatic compressibilities of transfer of some amino acids from water to aqueous lactose solutions at different temperatures. J. Mol. Liq., 162: 38-44.

DOI: 10.1016/j.molliq.2011.05.017

Palani, R. and A. Geeta, 2007. Acoustical and thermodynamical studies of 1-serine, 1-glutamine and l-asparagine in aqueous d-glucose solutions at 298.15 K. Res. J. Phys., 1: 82-89.

Palani, R. and K. Jayachitra, 2008. Ultrasonic study of ternary electrolytic mixtures at 303, 308 and $313 \mathrm{~K}$. Indian J. Pure Applied Phys., 46: 251-254.

Palani, R., S. Balakrishnan and G. Arumugam, 2011. Short communication ultrasonic studies of amino acids in aqueous sucrose solution at different temperatures. J. Phys. Sci., 22: 131-131.

Palani, R., S. Balakrishnan and R. Ashok Kumar, 2009. Ultrasonic studies of ion-solvent interactions of tetraalkylammoniumbromide in mixed solvent of DMF with propylene carbonate mixtures at 303.15K. J. Chem. Pharm. Sci., 2: 98-102.

Parmar, M.L. and R.C. Thakur, 2006. Effect of temperature on the viscosities of some divalent transition metal sulphates and magnesium sulphate in water and water + ethylene glycol mixtures. Indian J. Chem., 45A: 1631-1637.

Passynski, A., 1938. Acta Physico Chim., 8: 357-357.

Pfeiffer, C.C. and E.R. Braverman, 1982. Zinc, the brain and behavior. Biol. Psychiatry, 17: 513-532. PMID: 7082716

Pinto, R.R., D. Santos and S. Mattedietal, 2015. Density, refractive index, apparent volumes and excess molar volumes of four protic ionic liquids + water at $\mathrm{T}=298.15$ and $323.15 \mathrm{~K}$. Brazilian J. Chem. Eng., 32: 671-671. 
Rao, N.P. and R.E. Verrall, 1987. Ultrasonic velocity, excess adiabatic compressibility, apparent molar volume and apparent molar compressibility properties of binary liquid mixtures containing 2butoxyethanol. Can. J. Chem., 65: 810-816. DOI: $10.1139 / \mathrm{v} 87-137$

Rathore, P. and M. Singh, 2006. Registration of 'Ayiyi' Cowpea. Indian J. Chem., 45: 2650-2650. DOI: $10.2135 /$ cropsci2004.0766

Santosh, M.S., A. Lyubartsev, A. Mirzoev and D.K. Bhat, 2011. Solute-solvent interactions in aqueous glycylglycine- $\mathrm{CuCl}_{2}$ solutions: Acoustical and molecular dynamics perspective. J. Soln Chem., 40: 1657-1657. DOI: $10.1007 / \mathrm{s} 10953-011-9745-8$

Savaroglu, G. and M. Ozdemir, 2008. Apparent molar volume and apparent molar isentropic compressibility of glycerol in fructose-water at different temperatures. J. Mol. Liq., 137: 51-57. DOI: 10.1016/j.molliq.2007.03.007

Sawhney, N., M. Kumar, A.K. Sharma and M. Sharma, 2014. Ultrasonic studies on molecular interactions of $\mathrm{ZnSO}_{4}$ in aqueous solutions of glucose at various concentrations. J. Chem. Pharm. Res., 6: 1232-1242.

Singh, G. and T.S. Banipal, 2008. Partial molar adiabatic compressibilities and viscosities of some amino acids in aqueous glycerol solutions at $298.15 \mathrm{~K}$. Indian J. Chem., 47: 1355-1364.
Staiger, M.P. and A.M. Pietak, 2006. Magnesium and its alloys as orthopedic biomaterials: A review. Biomaterals, 27: 1728-1734.

DOI: $10.1016 /$ j.biomaterials.2005.10.003

Suryanarayan, C.V. and J. Kuppu Samy, 1981. J. Acoust. Soc. India.

Thirumaran, S. and A.N. Kannappan, 2009. Global J. Molecular Sci., 4: 160-160.

Timasheff, S.N. and T. Arakawa, 1989. Stabilization of Protein Structure by Solvents. In: Protein Structure: A Practical Approach, Creighton, T.E. (Ed.), IRL Press, New York, pp: 331-345.

Wadi, R.K. and P. Ramasami, 1997. Partial molal volumes and adiabatic compressibilities of transfer of glycine and dl-alanine from water to aqueous sodium sulfate at $288.15,298.15$ and 308.15 K. J. Chem. Soc. Faraday Trans., 93: 243-247. DOI: 10.1039/a604650i

Wapnir, R.A., 1990. Protein Nutrition and Mineral Absorption. 1st Edn., CRC Press, ISBN-13: 9780849352270, pp: 352. 